PROCEEDINGS OF THE

AMERICAN MATHEMATICAL SOCIETY

Volume 129 , Number 11, Pages 3221-3230

S 0002-9939(01)06165-2

Article electronically published on June 6, 2001

\title{
ROTATION NUMBERS IN THE INFINITE ANNULUS
}

\author{
PATRICE LE CALVEZ
}

(Communicated by Michael Handel)

\begin{abstract}
Using the notion of free transverse triangulation we prove that the rotation number of a given probability measure invariant by a homeomorphism of the open annulus depends continuously on the homeomorphism under some boundedness conditions.
\end{abstract}

\section{Notation}

We give the same name to the projections $p_{1}:(x, y) \mapsto x$ and $p_{2}:(x, y) \mapsto y$ defined on the infinite annulus $\mathbf{A}=\mathbf{T}^{1} \times \mathbf{R}$ or on the plane $\mathbf{R}^{2}$. We consider the projection

$$
\begin{aligned}
\pi: \mathbf{R}^{2} & \rightarrow \mathbf{A} \\
(x, y) & \mapsto(x+\mathbf{Z}, y)
\end{aligned}
$$

of the universal covering space $\mathbf{R}^{2}$ and the translation

$$
\begin{aligned}
T: \mathbf{R}^{2} & \rightarrow \mathbf{R}^{2} \\
(x, y) & \mapsto(x+1, y) .
\end{aligned}
$$

We will write $\mathbf{A}_{q}=\mathbf{R}^{2} / T^{q}$ for the finite covering space of order $q \geq 2$ of $\mathbf{A}$ and $\pi_{q}: \mathbf{A}_{q} \rightarrow \mathbf{A}$ for the projection.

We consider the set $H(\mathbf{A})$ of homeomorphisms of $\mathbf{A}$ homotopic to the identity and the set $h(\mathbf{A})$ of lifts to $\mathbf{R}^{2}$ of such maps. We will put on both sets the compactopen topology. If $\mu$ is a Borelian probability measure on $\mathbf{A}$, the set $H_{\mu}(\mathbf{A})$ of homeomorphisms $F \in H(\mathbf{A})$ which preserve $\mu$ is closed as is the set $h_{\mu}(\mathbf{A})$ of lifts.

If $E$ is a topological space, we will write respectively $\bar{X}$, $\operatorname{int}(X), \partial X$ for the closure, the interior and the frontier of $X \subset E$.

\section{INTRODUCTION}

If $F$ is a homeomorphism of the compact annulus $\mathbf{T}^{1} \times[0,1]$ homotopic to the identity and $f$ is a lift of $F$ to $\mathbf{R} \times[0,1]$, the rotation number of a point $z \in \mathbf{T}^{1} \times[0,1]$ is defined when it exists by the relation

$$
\rho(z)=\lim _{n \rightarrow \pm \infty} \frac{p_{1} \circ f^{n}(\widetilde{z})}{n}
$$

where $\widetilde{z} \in \pi^{-1}(\{z\})$.

Received by the editors February 23, 2000.

2000 Mathematics Subject Classification. Primary 37E30, 37E45.

(C)2001 American Mathematical Society 
If $F$ preserves a probability measure $\mu$, it is a direct consequence of the Birkhoff Ergodic Theorem that the rotation number is well defined $\mu$-a.e., that the measurable function $\rho$ obtained in this way is integrable and that $\int \rho d \mu=\int r d \mu$, where the map $r: \mathbf{A} \rightarrow \mathbf{R}$ is lifted to $\mathbf{R} \times[0,1]$ by $p_{1} \circ f-p_{1}$. This integral is called the rotation number of $\mu$. The rotation number is well defined for a homeomorphism $F$ of an abstract compact annulus if we choose a generator of the first homology group. Indeed, if $G$ is a homeomorphism of $\mathbf{T}^{1} \times[0,1]$ which induces the identity on the first homology group and $g$ is a lift of $G$ to $\mathbf{R} \times[0,1]$, it is easy to see that a point $z$ has a rotation number for $f$ iff $G(z)$ has a rotation number for $g \circ f \circ g^{-1}$ and that these numbers are equal. Moreover, if $\mu$ is an invariant measure on $\mathbf{A}$, the rotation number of $\mu$ for $f$ is equal to the rotation number of $G_{*}(\mu)$ for $g \circ f \circ g^{-1}$. The notion of rotation number is very classical and was introduced in the case of a general compact manifold by S. Schwartzman $[\mathrm{S}]$ as an element of the first group of homology.

In the case of a homeomorphism $F$ of the open annulus, the situation is more complicated. First of all, the existence of a rotation number for a point is not stable by conjugacy. Indeed, if $z$ has a rotation number (in the sense given above) and if the sequence $\left(u_{n}\right)_{n \geq 0}$ defined by $u_{n}=p_{2} \circ F^{n}(z)$ is unbounded, we can extract a strictly monotone unbounded subsequence $\left(u_{n_{k}}\right)_{k \geq 0}$. If we consider the homeomorphism

$$
\begin{aligned}
g: \mathbf{R}^{2} & \rightarrow \mathbf{R}^{2} \\
(x, y) & \mapsto(x+a(y), y)
\end{aligned}
$$

where $a: \mathbf{R} \rightarrow \mathbf{R}$ is continous and satisfies

$$
a\left(u_{n_{k}}\right)=-p_{1} \circ f^{n_{k}}(\widetilde{z})+p_{1}(\widetilde{z})+k n_{k}, \quad \widetilde{z} \in \pi^{-1}(\{z\}),
$$

and the element $G \in H(\mathbf{A})$ lifted by $g$, then the point $G(z)$ has no rotation number for $g \circ f \circ g^{-1}$. A natural way to define the rotation number of a point in that case is to limit ourselves to the recurrent points of $F$. We will say that a recurrent point $z$ has a rotation number $\rho(z)$ if for every subsequence $\left(F^{n_{k}}(z)\right)_{k \geq 0}$ of $\left(F^{n}(z)\right)_{n \geq 0}$ which converges to $z$ we have

$$
\lim _{k \rightarrow+\infty} \frac{p_{1} \circ f^{n_{k}}(\widetilde{z})}{n_{k}}=\rho(z)
$$

for every $\widetilde{z} \in \pi^{-1}(z)$ and if we have a similar result for the subsequences of $\left(F^{n}(z)\right)_{n \leq 0}$ converging to $z$. For this definition it becomes clear that this notion is stable by conjugacy.

If $F$ preserves a probability measure $\mu$ and if $r$ is $\mu$-integrable, we will get the same situation as in the compact case: $\mu$-a.e. point has a rotation number (in the strong sense) and we will get the rotation number of the measure by integrating this function. It may happen that after a conjugacy the map $r^{\prime}$ lifted by $p_{1} \circ g \circ f \circ g^{-1}-p_{1}$ is no longer integrable. However, if it is the case, the rotation number of the measure $G_{*}(\mu)$ for $g \circ f \circ g^{-1}$ is equal to $\int r^{\prime} d \mu$. Indeed $\mu$-a.e. point is recurrent for $F$ and the rotation number in the weak sense is invariant by conjugacy and coincides with the rotation number in the strong sense. So it is natural to say that a measure $\mu$ has a rotation number if $\mu$-a.e. recurrent point has a rotation number in the weak sense and if the measurable function $\rho$ obtained is integrable: the rotation number will be $\int \rho d \mu$. We have the following immediate properties: 
i) If $f^{\prime}=T^{k} \circ f$ is another lift of $F$, the rotation number of a point (or an invariant measure) for $f^{\prime}$ is obtained by adding $k$ to the rotation number for $f$.

ii) Fix $q \geq 2$ and consider the homeomorphism $F_{q}$ of $\mathbf{A}_{q}$ lifted by $f$. If $z$ is a recurrent point of $F_{q}$ and if its image in $\mathbf{A}$, which is a recurrent point of $F$, has a rotation number $\rho$, then $z$ has a rotation number equal to $\rho / q$ (we use the natural generator of $H_{1}\left(A_{q}, \mathbf{R}\right)$ defined by the translation $\left.T^{q}\right)$. So if an invariant measure $\mu$ of $F$ has a rotation number $\rho$ and if $\mu_{q}$ is the natural probability measure defined on $\mathbf{A}_{q}$ by $\mu$, there exists a set of full measure of $\mathbf{A}_{q}$ which consists of recurrent points of $F_{q}$ whose image in $\mathbf{A}$ has a rotation number. We deduce that $\mu_{q}$ has a rotation number which is equal to $\rho / q$.

There are examples of probability measures without rotation number. The homeomorphism $F \in H(\mathbf{A})$ lifted by

$$
\begin{aligned}
f: \mathbf{R}^{2} & \rightarrow \mathbf{R}^{2} \\
(x, y) & \mapsto(x+a(y), y)
\end{aligned}
$$

preserves the volume form $\frac{1}{\pi\left(1+y^{2}\right)} d x \wedge d y$ if $a$ is $C^{1}$. If $a$ is equal to $(-1)^{k} k^{2}$ on any interval $[k, k+1 / 2]$, the measure defined by the 2 -form has no rotation number.

If $z$ is a fixed point of $F$, there exists an integer $p \in \mathbf{Z}$ such that $f(z)=T^{p}(z)$. This integer is the rotation number of the point $z$ and the rotation number of the Dirac measure concentrated on $z$. In the previous example there were a lot of fixed points with rotation numbers arbitrarily large. In fact J. Franks [Fr2] proved that this is always the case when the measure has no rotation number and a support equal to $\mathbf{A}$. We will recall the proof of this result (Theorem 1), expressing it in terms of free transverse triangulation (notion introduced by M. Flucher [Fl]). This will permit us to show a continuity result (Theorem 2) for the rotation number of a measure. It is easy to see that it is optimal because the compact-open topology permits us to perturb a given map by changing it arbitrarily outside of a compact set. We denote by $\operatorname{Fix}(F)$ the set of fixed points of $F$, by $R(f)$ the smallest interval of $\mathbf{Z}$ which contains all the rotation numbers of the fixed points of $F$ and by $|R(f)|$ the cardinality of $R(f)$.

Theorem 1. Let $\mu$ be a probability measure, $F$ an element of $H_{\mu}(\mathbf{A})$ and $f \in$ $h_{\mu}(\mathbf{A})$ a lift of $F$. If $R(f)$ is finite, then $\mu$-a.e. recurrent point of $F$ has a rotation number. Moreover if the support of $\mu$ is $\mathbf{A}$, the rotation numbers are bounded and the measure $\mu$ has a rotation number.

Theorem 2. Let $\mu$ be a probability measure whose support is $\mathbf{A}$. If $\left(F_{n}\right)_{n>0}$ is a sequence in $H_{\mu}(\mathbf{A})$ converging to $F \in H_{\mu}(\mathbf{A})$, if $\left(f_{n}\right)_{n \geq 0}$ is a sequence of lifts converging to a lift $f$ of $F$ and if the sequence $\left(\left|R\left(f_{n}\right)\right|\right)_{n \geq 0}$ is uniformly bounded, then $R(f)$ is finite and the sequence of rotation numbers of $\mu$ for $f_{n}$ converges to the rotation number of $\mu$ for $f$.

\section{FREE DISK CHAINS}

A free disk of a homeomorphism $F$ of a surface $S$ is a set homeomorphic to an open disk which doesn't meet its image by $F$. A free disk chain $C$ is given by a family $\left(U_{i}\right)_{1 \leq i \leq n}$ of free disks and a family $\left(p_{i}\right)_{1 \leq i \leq n-1}$ of positive integers such that:

i) two disks $U_{i}$ and $U_{j}$ are equal or disjoint;

ii) for every $i \in\{1, \ldots, n-1\}$ the set $F^{p_{i}}\left(U_{i}\right)$ meets $U_{i+1}$. 
We say that the chain goes from $U_{1}$ to $U_{n}$ and will define the length of the chain $C$ to be the integer $l(C)=\sum_{i=1}^{n-1} p_{i}$; we say that the chain is closed if $U_{1}=U_{n}$. If $\mathcal{D}$ is a family of disjoint free disks of $F$, a $\mathcal{D}$-chain is a chain of disks chosen in $\mathcal{D}$. We say that $\mathcal{D}$ is transitive if for every pair of disks $U_{1}$ and $U_{2}$ in $\mathcal{D}$ we can find a $\mathcal{D}$-chain from $U_{1}$ to $U_{2}$.

We have the following fundamental result, due to J. Franks [Fr1], which is a consequence of the Brouwer Lemma on Arcs of Translation.

Proposition 1. A fixed point free and orientation preserving homeomorphism of $\mathbf{R}^{2}$ has no closed free disk chain.

Let $F$ be a fixed point free homeomorphism of a surface $S$ and consider a triangulation of $S$. We can construct a subtriangulation sufficiently small such that the closure of any 2-cell is free. Such a triangulation will be called a free triangulation. If we perturb slightly a free triangulation we still get a free triangulation. We can do it in such a way that we have the following property: if $\gamma$ and $\gamma^{\prime}$ are two edges such that $F(\gamma)$ (resp. $\left.F^{-1}(\gamma)\right)$ meets $\gamma^{\prime}$, then $F(\gamma)$ (resp. $F^{-1}(\gamma)$ ) meets the two 2-cells adjacent to $\gamma^{\prime}$. Such a triangulation will be called a free transverse triangulation. If $\mu$ is a probability measure, we can perturb slightly a free transverse triangulation to get another free transverse triangulation such that each edge of the triangulation is a null-set. Such a triangulation will be called a full free transverse triangulation (for $\mu$ ). We will denote by the same letter a triangulation $\mathcal{D}$ and the family of 2-cells of this triangulation. We have the following result (see [F] or [LS] for more details).

Proposition 2. If $F$ is a fixed point free homeomorphism of a connected surface $S$ which preserves a probability measure $\mu$ whose support is $S$, then the family of 2 -cells of a free transverse triangulation is transitive.

Proof. Let $\mathcal{D}=\left(V_{\alpha}\right)_{\alpha \in A}$ be the family of 2-cells of this triangulation. Fix $\alpha_{0} \in A$ and consider the set $A^{\prime}$ of elements $\alpha \in A$ such that there exists a $\mathcal{D}$-chain from $V_{\alpha_{0}}$ to $V_{\alpha}$. It is easy to prove that the set $W=\operatorname{int}\left(\overline{U_{\alpha \in A^{\prime}} V_{\alpha}}\right)$ is positively invariant and by the transversality condition that it satisfies $F(\partial W) \subset W$. From the fact that the support of the measure is $S$ we deduce that $\partial W=\emptyset$, and from the connectedness of $S$ we deduce that $W=S$ and that $A^{\prime}=A$.

Consider $F \in H(\mathbf{A})$, a lift $f \in h(\mathbf{A})$ and a family $\mathcal{D}$ of disjoint free disks of $F$, and write $\widetilde{\mathcal{D}}=\left(V_{\alpha}\right)_{\alpha \in A}$ the lifted family (i.e. the family of connected components of the preimages by $\pi$ of the disks) which consists of free disks of $f$. For $\alpha \in A$ and $k \in \mathbf{Z}$ we will write $\beta=\alpha+k$ if $V_{\beta}=T^{k}\left(V_{\alpha}\right)$.

For every $\alpha \in A$, the set $Z(\alpha)$ of integers $k \in \mathbf{Z}$ such that there exists a $\widetilde{\mathcal{D}}$-chain of $f$ from $V_{\alpha}$ to $V_{\alpha+k}$ is stable by addition. Indeed, if $k$ and $k^{\prime}$ belong to $Z(\alpha)$ we can concatenate a $\widetilde{\mathcal{D}}$-chain from $V_{\alpha}$ to $V_{\alpha+k}$ and a $\widetilde{\mathcal{D}}$-chain, translated by $T^{k}$, from $V_{\alpha}$ to $V_{\alpha+k^{\prime}}$ to get a $\widetilde{\mathcal{D}}$-chain from $V_{\alpha}$ to $V_{\alpha+k+k^{\prime}}$. We write

$$
Z(\mathcal{D})=\bigcup_{\alpha \in A} Z(\alpha)
$$

For every $\widetilde{\mathcal{D}}$-chain $C$ from $V_{\alpha}$ to a translated disk $V_{\alpha+k}$, consider the rational number $k / l(C)$, where $l(C)$ is the length of $C$. Write $Q(\alpha)$ for the set of rational numbers obtained in this way. If we change the lift $f$ by the lift $T^{s} \circ f$ we change 
the set $Q(\alpha)$ by $Q(\alpha)+s$. We define

$$
Q(\mathcal{D})=\bigcup_{\alpha \in A} Q(\alpha)
$$

If $f$ is fixed point free, the set $Z(\alpha)$ doesn't contain 0 by Proposition 1: we deduce that all the integers $k \in Z(\alpha)$ have the same sign using the additive property. If $F$ is fixed point free, for every $k \in \mathbf{Z}$, all the rational numbers in $Q(\alpha)+k$ have the same sign: we deduce that there exists an integer $m$ such that $Q(\alpha) \subset] m, m+1[$.

Suppose now that $\mathcal{D}$ is transitive and fix $\alpha_{1}$ and $\alpha_{2}$ in $A$. By the transitivity of $\mathcal{D}$ we know that there exist two integers $l_{1}$ and $l_{2}$, a $\widetilde{\mathcal{D}}$-chain from $V_{\alpha_{1}}$ to $V_{\alpha_{2}+l_{2}}$ and a $\widetilde{\mathcal{D}}$-chain from $V_{\alpha_{2}}$ to $V_{\alpha_{1}+l_{1}}$. Using concatenations and translations we deduce that for every $n \geq 0$ and for every $k_{1} \in Z\left(\alpha_{1}\right)$ the number $l_{2}+n k_{1}+l_{1}$ belongs to $Z\left(\alpha_{2}\right)$. If $f$ is fixed point free we deduce that all the integers $k \in Z(\mathcal{D})$ have the same sign, and if $F$ is fixed point free that there exists an integer $m$ such that $Q(\mathcal{D}) \subset] m, m+1[$.

Proposition 3. Fix $F \in H_{\mu}(\mathbf{A})$ and a lift $f \in h_{\mu}(\mathbf{A})$, where $\mu$ is a probability measure whose support is $\mathbf{A}$. Consider a free triangulation $\mathcal{D}$ of $\mathbf{A} \backslash \operatorname{Fix}(F)$ and a finite set $X \subset Q(\mathcal{D})$. If $f^{\prime} \in h_{\mu}(\mathbf{A})$ is sufficiently close to $f$, there exists a free transverse triangulation $\mathcal{D}^{\prime}$ of $\mathbf{A} \backslash \operatorname{Fix}\left(F^{\prime}\right)$, where $F^{\prime} \in H_{\mu}(\mathbf{A})$ is lifted by $f^{\prime}$, such that $X \subset Q\left(\mathcal{D}^{\prime}\right)$. Moreover if $X$ contains a nonnegative number and a nonpositive number, $f^{\prime}$ has a fixed point.

Proof. There exists a finite subset $\mathcal{D}_{1}$ of $\mathcal{D}$ such that $X \subset Q\left(\mathcal{D}_{1}\right)$. If $f^{\prime}$ is sufficiently close to $f$, the closure of every disk of $\mathcal{D}_{1}$ is free (for $F^{\prime}$ ) and we have the same relation $X \subset Q\left(\mathcal{D}_{1}\right)$ for $f^{\prime}$. We can complete this finite family to construct a free triangulation of $\mathbf{A} \backslash \operatorname{Fix}\left(F^{\prime}\right)$. If we perturb it slightly we will get a free transverse triangulation $\mathcal{D}^{\prime}$ of $\mathbf{A} \backslash \operatorname{Fix}\left(F^{\prime}\right)$ such that $X \subset Q\left(\mathcal{D}^{\prime}\right)$.

For any integer $q \geq 1$, denote by $\operatorname{Fix}_{\geq q} F$ the set of fixed points of $F$ whose rotation number has an absolute value larger than $q$. Consider $M>0$ such that the disks of $\mathcal{D}_{1}$ are contained in $\left.\mathbf{T}^{1} \times\right]-M, M[$ and such that this annulus meets its image by $F$. Find an integer $q \geq 1$ which bounds strictly the rotation numbers of the fixed points of $F$ which are in $\mathbf{T}^{1} \times[-M, M]$. If $f^{\prime} \in h_{\mu}(\mathbf{A})$ is sufficiently close to $f$ and if $F^{\prime} \in H_{\mu}(\mathbf{A})$ is lifted by $f^{\prime}$, the set $\left.\mathbf{T}^{1} \times\right]-M, M[$ meets its image by $F^{\prime}$ and $q$ bounds strictly the rotation numbers of the fixed points of $F^{\prime}$ which are in $\mathbf{T}^{1} \times[-M, M]$. If $f^{\prime}$ has no fixed point, the disks of the family $\mathcal{D}_{1}^{q}$ obtained by lifting $\mathcal{D}_{1}$ to $\mathbf{A}_{q}$ are contained in the same connected component $W^{\prime}$ of $\mathbf{A}_{q} \backslash \pi_{q}^{-1}\left(\operatorname{Fix}\left(F_{\geq q}^{\prime}\right)\right)$ and this component is invariant by the lift of $F^{\prime}$. Moreover the set $Q\left(\mathcal{D}_{1}^{q}\right)$ contains a nonnegative and a nonpositive number. Completing $\mathcal{D}_{1}^{q}$ and perturbing it we can find a free transverse triangulation $\mathcal{D}^{\prime}$ of $W^{\prime}$ such that $Q\left(\mathcal{D}^{\prime}\right)$ has the same property. Since the triangulation $\mathcal{D}^{\prime}$ is transitive by Proposition 2, the map $f^{\prime}$ must have a fixed point.

Corollary 1. Fix $F \in H_{\mu}(\mathbf{A})$ and a lift $f \in h_{\mu}(\mathbf{A})$, where $\mu$ is a probability measure whose support is $\mathbf{A}$. If there exist two recurrent points $z_{1}$ and $z_{2}$ with rotation numbers $\rho\left(z_{1}\right)$ and $\rho\left(z_{2}\right)$ such that $\rho\left(z_{1}\right)<0<\rho\left(z_{2}\right)$, then $f$ has a fixed point. Moreover every $f^{\prime} \in h_{\mu}(\mathbf{A})$ sufficiently close to $f$ has a fixed point.

Proof. If these points are not fixed by $F$ we can find a free transverse triangulation $\mathcal{D}$ of $\mathbf{A} \backslash \operatorname{Fix}(F)$ such that $z_{1}$ and $z_{2}$ belong to 2-cells. The hypothesis tells us that $Q(\mathcal{D})$ contains a strictly negative and a strictly positive number and 
we can apply Proposition 3. If at least one of them is fixed we can take an integer $q>\max \left(\left|\rho\left(z_{1}\right)\right|,\left|\rho\left(z_{2}\right)\right|\right)$ and consider a free transverse triangulation $\mathcal{D}$ of $\mathbf{A}_{q} \backslash \pi_{q}^{-1}\left(\operatorname{Fix}\left(F_{\geq q}\right)\right)$ such that every preimage of $z_{1}$ or $z_{2}$ belongs to a disk of $\mathcal{D}$. We will get the same situation as above.

Corollary 2. Let $\left(F_{n}\right)_{n \geq 0}$ be a sequence in $H_{\mu}(\mathbf{A})$ which converges to $F \in H_{\mu}(\mathbf{A})$ and $\left(f_{n}\right)_{n \geq 0}$ a sequence of lifts in $h_{\mu}(\mathbf{A})$ which converges to $f \in h_{\mu}(\mathbf{A})$, where $\mu$ is a probability measure whose support is $\mathbf{A}$. If the integers $\left|R\left(f_{n}\right)\right|$ are uniformly bounded, then $R(f)$ is finite and there exists a uniform bound to the rotation numbers of the recurrent points of the $F_{n}$.

Proof. Corollary 1, applied to a translation of $f$ or $f_{n}$, tells us that $R(f)$ and $R\left(f_{n}\right)$ are intervals of $\mathbf{Z}$. Moreover, if $\rho^{-} \in R(f)$ and $\rho^{+} \in R(f)$ satisfy $\rho^{+}-\rho^{-} \geq 2$, then every integer $\rho \in\left[\rho^{-}+1, \rho^{+}-1\right]$ belongs to $R\left(f_{n}\right)$ for $n$ large enough. We deduce that $R(f)$ is finite and that the set of rotation numbers of recurrent points of $F$ is bounded. For the same reasons the set of rotation numbers of the recurrent points of each $F_{n}$ is bounded. We want to find a uniform bound; it is sufficient to find it for $n$ large enough.

Define $M=\max _{n}\left|R\left(f_{n}\right)\right| \geq 0$ and consider $q=M+2$. We can find an interval $I \in \mathbf{Z}$ of cardinality $M+1$ such that if $k \in I$, the map $F^{\prime}$ of $\mathbf{A}_{q}$ lifted by $f^{\prime}=T^{-k} \circ f$ is different from the identity. If $F$ is different from the identity, any choice is good; if $F$ is the identity, there exists $p$ such that $T^{-p} \circ f$ is the identity and we choose $I$ disjoint from $p+q \mathbf{Z}$. For every $n \geq 0$, there exists $k \in I$ such that $R\left(f_{n}\right)$ doesn't meet $I$. That means that the homeomorphism $F_{n}^{\prime}$ of $\mathbf{A}_{q}$ lifted by $f_{n}^{\prime}=T^{-k} \circ f_{n}$ is fixed point free. To prove the corollary it is sufficient to fix $k \in I$ and to show that the rotation numbers of the recurrent points of $F_{n}$ are uniformly bounded if $F_{n}^{\prime}$ is fixed point free. Consider a free transverse triangulation $\mathcal{D}$ of $\mathbf{A}_{q} \backslash \operatorname{Fix}\left(F^{\prime}\right)$ and the set $\mathcal{Q}(\mathcal{D})$ defined for the lift $f^{\prime}$ or $F^{\prime}$, fix a number $\rho \in Q(\mathcal{D})$ and take a finite sub-family $\mathcal{D}_{1}$ such that $\rho \in Q\left(\mathcal{D}_{1}\right)$. If $n$ is large enough, then $\mathcal{D}_{1}$ is a family of disks whose closure is free for $F_{n}^{\prime}$. If $z$ is a recurrent point of $F_{n}$ of rotation number $\rho^{\prime}$, then by extending the family $\mathcal{D}_{1}$ and perturbing it we can construct a free transverse triangulation $\mathcal{D}$ of $\mathbf{A}_{q}$ for $F_{n}^{\prime}$ such that $\rho \in Q(\mathcal{D})$ and such that every preimage of $z$ in $\mathbf{A}_{q}$ belongs to a disk of $\mathcal{D}$. We deduce that we can find in $Q(\mathcal{D})$ rational numbers arbitrarily close to $\rho^{\prime} / q$. This implies that $\rho^{\prime} / q \in[\rho-1, \rho+1]$ because $F_{n}^{\prime}$ is fixed point free.

\section{Proofs of THE THEOREMS}

Proof of Theorem 1. We consider a probability measure $\mu$ on $\mathbf{A}$, a homeomorphism $F \in H_{\mu}(\mathbf{A})$ and a lift $f \in h_{\mu}(\mathbf{A})$. We suppose that the set $R(f)$ is bounded. Every fixed point of $F$ has a rotation number and the set of nonfixed points of $F$ can be covered by free disks. So it is sufficient to prove that if $U$ is a free disk of $F$ such that $\mu(U)>0$, then $\mu$-a.e. recurrent point of $F$ in $U$ has a rotation number.

We denote by $\Phi$ the first return map defined a.e. on $U$ and by $\nu$ the time of first return. The measurable function $\nu$ is integrable on $U$ and we have

$$
\int_{U} \nu d \mu=\mu\left(\bigcup_{k \geq 0} F^{k}(U)\right) .
$$


Indeed, we have the following measurable partitions (modulo sets of measure zero):

$$
U=\bigsqcup_{i \geq 1} U_{i} \text { and } \bigcup_{k \geq 0} F^{k}(U)=\bigsqcup_{i \geq 1} \bigsqcup_{0 \leq j \leq i-1} F^{j}\left(U_{i}\right)
$$

where $U_{i}=\nu^{-1}(\{i\})$; therefore

$$
\mu\left(\bigcup_{k \geq 0} F^{k}(U)\right)=\sum_{i \geq 1} \sum_{0 \leq j \leq i-1} \mu\left(U_{i}\right)=\sum_{i \geq 1} i \mu\left(U_{i}\right)=\int_{U} \nu d \mu .
$$

Fix a connected component $\widetilde{U}$ of $\pi^{-1}(U)$ and consider $\tau: U \rightarrow \mathbf{Z}$ such that

$$
\tau(z)=k \quad \text { iff } \quad f^{\nu(z)}(\widetilde{z}) \in T^{k}(\widetilde{U}),
$$

where $\widetilde{z}$ is the preimage of $z$ contained in $\widetilde{U}$.

Using the fact that the disk $U$, taken alone, defines a transitive family of free disks of $F$ and using the properties of the sets $Q(\alpha)$ stated in Section 2, we deduce that the function $\tau / \nu$ is bounded. More precisely we recall that there exists a fixed point of $f \circ T^{-k}$ for every integer $k$ surrounded by two points in the image of $\tau / \nu$.

Since the function $\nu$ is integrable, it is the same for $\tau$ and we know by the Poincaré Recurrence Theorem and the Birkhoff Ergodic Theorem that almost every point of $U$ satisfies the following properties:

i) $z$ is a recurrent point of $\Phi$;

ii) $\lim _{n \rightarrow \pm \infty} \frac{\tau(z)+\cdots+\tau\left(\Phi^{n-1}(z)\right)}{n}=\tau^{*}(z)$ exists;

ii) $\lim _{n \rightarrow \pm \infty} \frac{\nu(z)+\cdots+\nu\left(\Phi^{n-1}(z)\right)}{n}=\nu^{*}(z)$ exists.

The recurrent points of $F$ are exactly the recurrent points of $\Phi$ because $U$ is open. We deduce that almost every recurrent point of $F$ in $U$ has a rotation number which is

$$
\lim _{n \rightarrow \pm \infty} \frac{\tau(z)+\cdots+\tau\left(\Phi^{n-1}(z)\right)}{\nu(z)+\cdots+\nu\left(\Phi^{n-1}(z)\right)}=\frac{\tau^{*}(z)}{\nu^{*}(z)} .
$$

If the support of $\mu$ is $\mathbf{A}$ we know using the corollaries in Section 2 that the rotation numbers are uniformly bounded and that the measure has a rotation number.

Remark. If the support of $\mu$ is not $\mathbf{A}$ the second part is obviously false. Suppose that $f(x, k)=(x+k+1 / 2, k)$ if $k \in \mathbf{Z}$ and that $F$ is fixed point free. One can construct easily a discrete invariant measure without rotation number.

Proof of Theorem 2. We consider a probability measure $\mu$ on $\mathbf{A}$, a sequence $\left(F_{n}\right)_{n \geq 0}$ in $H_{\mu}(\mathbf{A})$ which converges to $F$ and a sequence of lifts $\left(f_{n}\right)_{n \geq 0}$ which converges to a lift $f$ of $F$. We suppose that the integers $\left|R\left(f_{n}\right)\right|$ are uniformly bounded. Corollary 2 tells us that $R(f)$ is bounded and that the rotation numbers of recurrent points of the $F_{n}$ are bounded independently of $n$. By Theorem 1 we can define the rotation number $\rho_{n}$ of $\mu$ for each $f_{n}, n \geq 0$, and the rotation number $\rho$ of $\mu$ for $f$. We know that the sequence $\left(\rho_{n}\right)_{n \geq 0}$ is bounded and want to prove that it converges to $\rho$. Consider an integer $k \geq 1$ such that the rotation numbers of recurrent points of each $F_{n}$ or $F$ are contained in $[-k, k]$. Taking $f_{n}^{\prime}=T^{k+1} \circ f_{n}$ instead of $f_{n}$ and $\mathbf{A}_{2 k+2}$ instead of $\mathbf{A}$ we can suppose that every $F_{n}$ (and $F$ ) is fixed point free and that every $\rho_{n}$ (and $\rho$ ) belongs to ]0,1[. Using the results of Section 2 we deduce that for any free transverse triangulation $\mathcal{D}$ of $\mathbf{A}$ for $F_{n}$ or $F$, we have $\left.Q(\mathcal{D}) \subset\right] 0,1[$. 
Indeed the set $Q(\mathcal{D})$ meets ]0,1[ because the rotation number of any recurrent point belongs to this interval and cannot meet $]-\infty, 0]$ or $[1, \infty[$ ), otherwise they would be a fixed point of $F\left(\right.$ or $\left.F_{n}\right)$.

Consider a full free transverse triangulation $\mathcal{D}=\left(V_{\alpha}\right)_{\alpha \in A}$ of $F$ such that every disk has a diameter $<1$. Then a finite subfamily $\mathcal{D}_{1}=\left(V_{\alpha}\right)_{\alpha \in A_{1}}$ of $\mathcal{D}$ such that the measure of $W=\operatorname{int}\left(\overline{\bigcup_{\alpha \in A_{1}} V_{\alpha}}\right)$ is larger than $1-\varepsilon$. By the transitivity of $\mathcal{D}$ we know that there exists a larger finite subfamily $\mathcal{D}_{2}=\left(V_{\alpha}\right)_{\alpha \in A_{2}}$ and $M_{1}>0$ such that for every $\alpha$ and $\alpha^{\prime}$ in $A_{1}$ there exists a $\mathcal{D}_{2}$-chain from $V_{\alpha}$ to $V_{\alpha^{\prime}}$ of length $\leq M_{1}$.

Let $\widetilde{\mathcal{D}}=\left(V_{\alpha}\right)_{\alpha \in \widetilde{A}}, \widetilde{\mathcal{D}}_{1}=\left(V_{\alpha}\right)_{\alpha \in \widetilde{A}_{1}}$ and $\widetilde{\mathcal{D}_{2}}=\left(V_{\alpha}\right)_{\alpha \in \widetilde{A}_{2}}$ be the lifted families. There exists $M_{2}>0$ such that for every $\alpha \in \widetilde{A}_{1}$ and $\alpha^{\prime} \in \widetilde{A}_{1}$ there is an integer $k \in \mathbf{Z}$ and a $\widetilde{\mathcal{D}_{2}}$-chain from $V_{\alpha}$ to $V_{\alpha^{\prime}+k}$ of length $\leq M_{1}$ and such that $V_{\alpha}$ and $V_{\alpha^{\prime}+k}$ can be projected by $p_{1}$ on an interval of length $\leq M_{2}$.

Consider the first return map $\Phi$ defined $\mu$-a.e. on $W$, the time of first return $\nu$, and the function

$$
\lambda: z \mapsto \sum_{i=0}^{\nu(z)-1} r\left(F^{i}(z)\right)=p_{1} \circ f^{\nu(z)}(\widetilde{z})-p_{1}(\widetilde{z}), \quad \widetilde{z} \in \pi^{-1}(\{z\}) .
$$

If $\Phi(z)$ is defined, using Baire's theorem, we can find $z^{\prime}$ close to $z$ such that

- every point $F^{i}\left(z^{\prime}\right), 0 \leq i \leq \nu(z)$, belongs to $V_{\alpha_{i}}, \alpha_{i} \in A$;

- $\alpha_{0}$ and $\alpha_{\nu(z)}$ are in $A_{1}$;

- $z \in \bar{V}_{\alpha_{0}}$ and $F(z) \in \bar{V}_{\alpha_{\nu(z)}}$.

If $\widetilde{z} \in \pi^{-1}(\{z\})$, we can lift the chain $\left(V_{\alpha_{i}}\right)_{0 \leq i \leq \nu(z)}$ to get a $\widetilde{\mathcal{D}}$-chain from $V_{\alpha}$ to $V_{\alpha^{\prime}}$ where

- $\alpha$ and $\alpha^{\prime}$ are in $\widetilde{A_{1}}$;

- $\widetilde{z} \in \bar{V}_{\alpha}$ and $f(\widetilde{z}) \in \bar{V}_{\alpha^{\prime}}$.

We can add to this chain a $\widetilde{\mathcal{D}}_{2}$-chain from $V_{\alpha^{\prime}}$ to a translated $V_{\alpha+k}$ to get a $\widetilde{\mathcal{D}}$-chain from $V_{\alpha}$ to $V_{\alpha+k}$ of length between $\nu(z)$ and $\nu(z)+M_{1}$. The fact that each disk has a diameter $<1$ and that $V_{\alpha}$ and $V_{\alpha+k}$ are projected in an interval of length $\leq M_{2}$ implies that $|k-\lambda(z)| \leq M_{2}+2$. The fact that $\left.Q(\mathcal{D}) \subset\right] 0,1[$ implies that $0<k<\nu(z)+M_{1}$. Therefore

$$
\begin{aligned}
& -\nu(z)\left(M_{1}+M_{2}+3\right)<-M_{2}-2<\lambda(z) \\
& \quad<\nu(z)+M_{1}+M_{2}+2 \leq \nu(z)\left(M_{1}+M_{2}+3\right)
\end{aligned}
$$

and

$$
\left|\frac{\lambda(z)}{\nu(z)}\right| \leq M_{1}+M_{2}+3=M_{3}
$$

As in the proof of Theorem 1, $\nu$ and $\lambda$ are integrable. From Theorem 1 we know that for $\mu$-a.e. point $z \in W$ :

i) $\quad z$ is a recurrent point of $\Phi$;

ii) $\lim _{n \rightarrow \pm \infty} \frac{\nu(z)+\cdots+\nu\left(\Phi^{n-1}(z)\right)}{n}=\nu^{*}(z)$ exists;

iii) $\lim _{n \rightarrow \pm \infty} \frac{\lambda(z)+\cdots+\lambda\left(\Phi^{n-1}(z)\right)}{n}=\lambda^{*}(z)$ exists;

iv) the rotation number $\rho(z)$ exists and we have $\rho(z)=\frac{\lambda^{*}(z)}{\nu^{*}(z)}$. 
Using the fact that $\rho$ is $F$-invariant, that $\mu$ is $\Phi$-invariant and the Birkhoff Ergodic Theorem we obtain

$$
\int_{\bigcup_{k \geq 0} F^{k}(W)} \rho d \mu=\int_{W} \nu \rho d \mu=\int_{W} \nu^{*} \rho d \mu=\int_{W} \lambda^{*} d \mu=\int_{W} \lambda d \mu .
$$

The first equality is obtained by arguing as in the proof of Theorem 1.

For $n$ large enough $\mathcal{D}_{2}$ is a family of free disks of $f_{n}$ and we have the same situation: we define $\Phi_{n}, \nu_{n}$ and $\lambda_{n}$ on $W$ with the same inequalities, we define $\rho_{n}$ on $\mathbf{A}, \nu_{n}^{*}$ and $\lambda_{n}^{*}$ on $W$ with the same equality.

We write

$$
\begin{aligned}
\left|\int_{\bigcup_{k \geq 0} F^{k}(W)} \rho d \mu-\int_{\bigcup_{k \geq 0} F_{n}^{k}(W)} \rho_{n} d \mu\right| & =\int_{W}\left(\lambda-\lambda_{n}\right) d \mu \\
& =\int_{W}\left(\frac{\lambda}{\nu}-\frac{\lambda_{n}}{\nu_{n}}\right) \nu d \mu+\int_{W} \frac{\lambda_{n}}{\nu_{n}}\left(\nu-\nu_{n}\right) d \mu .
\end{aligned}
$$

First we obtain

$$
\left|\int_{W} \frac{\lambda_{n}}{\nu_{n}}\left(\nu-\nu_{n}\right) d \mu\right| \leq \int_{W} M_{3}\left|\nu^{\prime}-\nu_{n}^{\prime}\right| d \mu \leq M_{3} \int_{W}\left(\nu^{\prime}+\nu_{n}^{\prime}\right) d \mu \leq 2 M_{3} \varepsilon,
$$

where $\nu^{\prime}=\nu-1$ and $\nu_{n}^{\prime}=\nu_{n}^{\prime}-1$. Then we write

$$
\left|\frac{\lambda}{\nu}-\frac{\lambda_{n}}{\nu_{n}}\right| \nu \leq 2 M_{3} \nu
$$

Using the fact that the boundary of $W$ has measure 0 (because the triangulation is full)) we deduce that the function $\frac{\lambda}{\nu}-\frac{\lambda_{n}}{\nu_{n}}$ tends to zero a.e. when $n \rightarrow+\infty$. So by the Lebesgue Dominated Convergence Theorem, for $n$ large enough we have

$$
\left|\int_{W}\left(\frac{\lambda}{\nu}-\frac{\lambda_{n}}{\nu_{n}}\right) \nu, d \mu\right| \leq \varepsilon
$$

Using the fact that all the rotation numbers belong to $[0,1]$ we deduce that

$$
\left|\int_{\mathbf{A}} \rho d \mu-\int_{\bigcup_{k \geq 0} F^{k}(W)} \rho d \mu\right| \leq \varepsilon
$$

and

$$
\left|\int_{\mathbf{A}} \rho_{n} d \mu-\int_{\bigcup_{k \geq 0} F_{n}^{k}(W)} \rho_{n} d \mu\right| \leq \varepsilon .
$$

We have obtained

$$
\left|\rho_{n}-\rho\right| \leq\left(2 M_{3}+3\right) \varepsilon
$$

for $n$ large enough, and the sequence $\left(\rho_{n}\right)_{n \geq 0}$ converges to $\rho$.

\section{REFERENCES}

[Fl] M. Flucher, Fixed points of measure preserving torus homeomorphisms, Manuscripta Math., 68 (1990), 271-293. MR 91j:58129

[Fr1] J. Franks, Generalizations of the Poincaré-Birkhoff theorem, Annals of Math., 128 (1988), 139-151. MR 89m:54052

[Fr2] J. Franks, Area preserving homeomorphisms of open surfaces of genus zero, New York J. Math., 2 (1996), 1-19. MR 97c:58123 
[LS] P. Le Calvez, A. Sauzet, Une démonstration dynamique du théorème de translation de Brouwer, Expo. Math., 14 (1996), 277-287. MR 97e:54043

[S] S. Schwartzman, Asymptotic cycles, Annals of Math., 68 (1957), 270-284. MR 19:568i

Laboratoire Analyse, Géométrie et Applications, UMR CNRS 7539, Institut Galilée, Université Paris Nord, 93430 Villetaneuse, France

E-mail address: lecalvez@math.univ-paris13.fr 